\title{
Tools for 'safety netting' in common paediatric illnesses: a systematic review in emergency care
}

\author{
Evelien de Vos-Kerkhof, ${ }^{1}$ Dorien HF Geurts, ${ }^{1}$ Mariska Wiggers, ${ }^{2}$ Henriette A Moll, ${ }^{1}$ \\ Rianne Oostenbrink ${ }^{1}$
}

- Additional material is published online only. To view please visit the journal online (http://dx.doi.org/10.1136/ archdischild-2014-306953).

1 Department of General Paediatrics, ErasmusMC-Sophia Children's Hospital, Rotterdam, The Netherlands

${ }^{2}$ Erasmus University, Rotterdam, The Netherlands

\section{Correspondence to}

Dr Rianne Oostenbrink, Department of General Paediatrics, ErasmusMC-Sophia Children's Hospital, Room Sp-1549, Wytemaweg 80, Rotterdam 3015 CN, The Netherlands; r.oostenbrink@erasmusmc.n

Received 11 June 2014 Revised 9 June 2015 Accepted 17 June 2015 Published Online First 10 July 2015

\section{CrossMark}

\author{
To cite: de Vos-Kerkhof $E_{\text {, }}$ \\ Geurts DHF, Wiggers M, \\ et al. Arch Dis Child \\ 2016;101:131-139.
}

\section{ABSTRACT}

Context Follow-up strategies after emergency department (ED) discharge, alias safety netting, is often based on the gut feeling of the attending physician. Objective To systematically identify evaluated safetynetting strategies after ED discharge and to describe determinants of paediatric ED revisits.

Data sources MEDLINE, Embase, CINAHL, Cochrane central, OvidSP, Web of Science, Google Scholar, PubMed.

Study selection Studies of any design reporting on safety netting/follow-up after ED discharge and/or determinants of ED revisits for the total paediatric population or specifically for children with fever, dyspnoea and/or gastroenteritis. Outcomes included complicated course of disease after initial ED visit (eg, revisits, hospitalisation).

Data extraction Two reviewers independently assessed studies for eligibility and study quality. As meta-analysis was not possible due to heterogeneity of studies, we performed a narrative synthesis of study results. A best-evidence synthesis was used to identify the level of evidence.

Results We summarised 58 studies, 36\% (21/58) were assessed as having low risk of bias. Limited evidence was observed for different strategies of safety netting, with educational interventions being mostly studied. Young children, a relevant medical history, infectious/ respiratory symptoms or seizures and progression/ persistence of symptoms were strongly associated with ED revisits. Gender, emergency crowding, physicians' characteristics and diagnostic tests and/or therapeutic interventions at the index visit were not associated with revisits.

Conclusions Within the heterogeneous available evidence, we identified a set of strong determinants of revisits that identify high-risk groups in need for safety netting in paediatric emergency care being related to age and clinical symptoms. Gaps remain on intervention studies concerning specific application of a uniform safety-netting strategy and its included time frame.

\section{INTRODUCTION}

When patients are discharged from the emergency department (ED) without definite diagnosis, monitoring children's course of disease to rule out serious infections is mandatory. ${ }^{1}$ This theme is covered by the term 'safety netting', introduced to general practice in 2004 by Roger Neighbour who considered it a core component of general practice consultation. ${ }^{2}$ Safety netting can be described as a set of procedures or guidelines, which should be followed when a patient is discharged from the ED. This strategy is

\section{What is already known on this topic}

The importance of safety netting after emergency department (ED) discharge to monitor disease course is well recognised and applied.

- Evidence-based approaches on this topic are underexposed in literature, since strategies are often based on the gut feeling of the ED physician.

\section{What this study adds}

- It remains difficult to determine high-risk patient groups for whom safety netting is essential.

- Studies concerning follow-up were mostly conflicting or with limited evidence.

- Young children, medical history, infectious/ respiratory symptoms, seizures and progression/ persistence of symptoms were the strongest associated determinants of revisits.

required in situations with increased risk for serious complications, either in the diagnosis itself (eg, dehydration in patients with gastroenteritis) or if individual patient characteristics are associated with a high risk of complications (eg, significant comorbidity or immunosuppressive therapy). ${ }^{1}$ Patients who revisit the ED may be regarded as the high-risk population of possible failure of this safety-netting strategy.

The importance of safety netting is increasingly recognised in emergency care and literature. ${ }^{3}$ Healthcare physicians lack standardised safetynetting methods since strategies are often based on the gut feeling of the ED physician, ${ }^{4}$ and key gaps are described in need of studies on methods and effects of safety netting. ${ }^{3}{ }^{5}$ Therefore, we planned to systematically review the literature on this important topic.

Our first aim was to systematically summarise evaluated safety-netting strategies after ED discharge. Second, we identified children at risk for revisits to improve the identification of children prone to deteriorate after emergency discharge, by studying determinants of ED revisits. Both aims were studied in the total ED population or specifically for children with common illnesses as fever, dyspnoea and gastroenteritis. 


\section{METHODS}

\section{Inclusion criteria}

We considered all types of studies eligible if they reported about safety netting and/or their strategy after ED discharge and extended our search for determinants of ED revisits as a proxy of failing safety-netting strategies. We included studies on the total ED population or specific for children with fever, dyspnoea and gastroenteritis. Studies reporting data on adult and children together as well as studies in low-income countries, due to differences in healthcare organisation, were excluded. Two reviewers independently assessed inclusion (EdV-K and MW); discrepancies were resolved by a third reviewer $(\mathrm{RO})$.

\section{Outcome measures}

Outcomes included complicated course of disease after initial ED visit, mainly dominated by revisits and hospitalisation.

\section{Search strategy}

We searched the following electronic databases: MEDLINE OvidSP, Embase (Excerpta Medica dataBASE), CINAHL (Cumulative Index to Nursing and Allied Health Literature), Cochrane central register of controlled trials, Web of Science, Google Scholar and PubMed as publisher (searches updated in January 2014) (see online supplementary information 1). We checked the reference list of these papers for additional articles that were not included in the initial computerised search.

\section{Data extraction}

We retrieved the full text copies of all articles identified as potentially relevant by reviewing the abstracts of search results. Two reviewers' extracted data on the following: study design, disease/working diagnosis, study population, number of revisits, follow-up period and type of revisit. The determinants were grouped into: child characteristics, social/demographics, disease characteristics, physician and process characteristics. Finally, data on follow-up after ED discharge, including the follow-up strategy, were extracted.

\section{Risk-of-bias assessment and best-evidence synthesis}

Two authors (EdV-K/DHFG) independently assessed the potential risk of bias of the studies included using the MINORS, a methodological index for non-randomised studies, ${ }^{6}$ together with the presence of revisits as primary outcome measure and the number of events (see online supplementary information 2). Consensus was reached by the two reviewers (EdV-K/DHFG); otherwise, the independent opinion of a third reviewer was decisive (RO).

We performed two separate 'best-evidence' syntheses based on the study of van Tulder et al; ${ }^{7}$ one according to safetynetting strategies and one according to determinants of revisits as meta-analysis of results was not possible owing to heterogeneity in participants, interventions, outcome measures and methodological quality $^{7}$ (see online supplementary information 2 ).

\section{RESULTS}

Identification and selection of the literature

The literature search identified 2604 references (figure 1). Overall, 36 of 83 full text articles screened for eligibility were excluded on the basis of incorrect study aims, data on adult patients, reviews or low-income populations. Data extraction was performed for 58 articles, including 11 articles added from reference lists. Forty two articles described determinants of

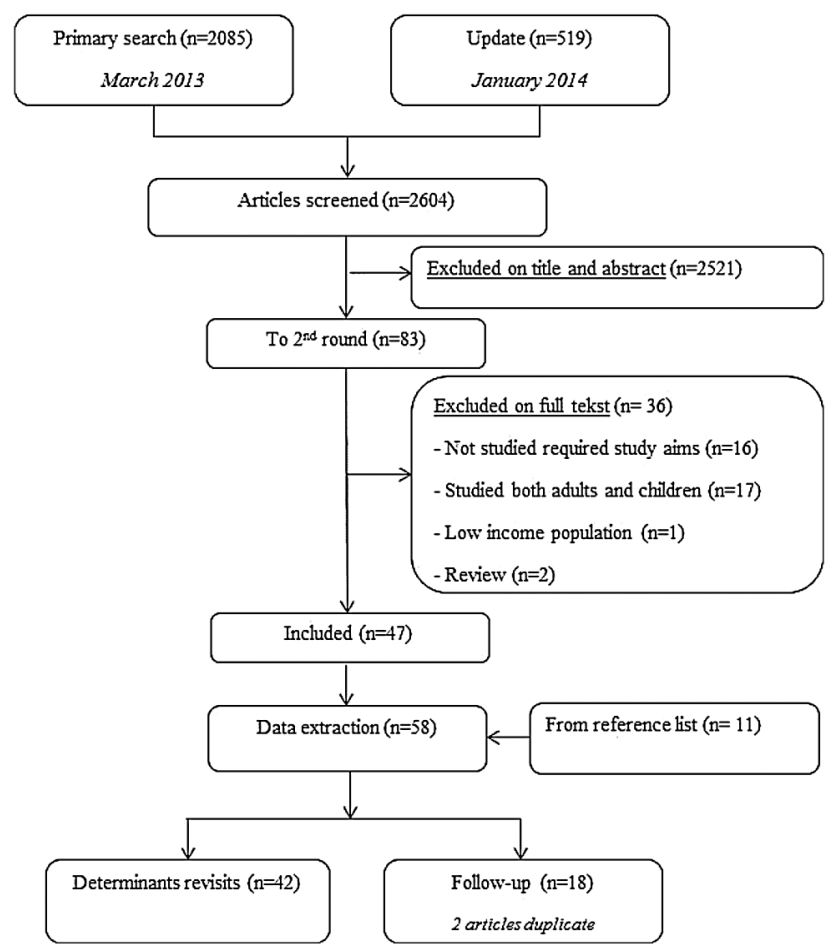

Figure 1 Flowchart of the study selection and exclusion stages during the systematic review process.

revisits, and 18 articles (2 articles duplicate) reported on follow-up after ED discharge (figure 1).

\section{Description of included studies}

Study characteristics are presented in tables 1 and 2 . Included studies were mostly cohort studies $(72 \%, \mathrm{n}=42)$. Fifty two per cent $(n=30)$ of the studies originated from the USA and 19\% $(n=11)$ from the UK. Year of publication varied between 1995 and 2013, with 33\% $(\mathrm{n}=19)$ published in the last 2 years. Most studies $(n=34)$ included all children presented to the ED or the most common paediatric illnesses; 14 studied febrile children and 10 studies reported specific diseases only (eg, gastroenteritis, influenza, respiratory tract infections). Study populations varied between 13 and 568845 children (median: $n=1371$ ) and number of events (revisits or hospitalisation after revisit) varied between 9 and 36734 (median: $n=189$ ). Follow-up period after ED discharge varied between 1 and 656 days (median: 3 days). Most studies $(n=29,50 \%)$ described scheduled and unscheduled revisits together; 19 (33\%) only measured unscheduled revisits (tables 1 and 2).

\section{Risk-of-bias assessment}

Online supplementary information 2 shows the potential risk of bias with $36 \%(n=21)$ of the studies having low risk of bias. For all studies, the reviewers achieved uniform bias assessment. Ten studies (17\%) were scored as high risk of bias because only abstracts were available (nine Congress abstracts and one Spanish abstract). Initial disagreement on 55 out of 880 assessed items $(6 \%)$ for opportunity of bias was solved by consensus reached by the two reviewers (EdV-K/DHFG) or by the decision of a third reviewer ( $\mathrm{RO})$.

\section{Safety netting after discharge}

Figure 2 presents an overview of the different safety-netting strategies evaluated in the included studies $(n=18)$ and the 
Table 1 Characteristics of included studies regarding the first study aim: safety-netting strategies after emergency department (ED) discharge

\begin{tabular}{|c|c|c|c|c|c|c|c|c|c|c|}
\hline $\begin{array}{l}\text { Author } \\
\text { Year } \\
\text { Country }\end{array}$ & $\begin{array}{l}\text { Study } \\
\text { design }\end{array}$ & $\begin{array}{l}\text { Article/ } \\
\text { abstract }\end{array}$ & $\begin{array}{l}\text { Disease/ } \\
\text { working } \\
\text { diagnosis }\end{array}$ & $\begin{array}{l}\text { Primary } \\
\text { outcome: } \\
\text { revisits }\end{array}$ & $\begin{array}{l}\mathrm{N} \text { total, } \\
\text { male } \%\end{array}$ & $\begin{array}{l}\mathrm{N} \\
\text { outcome, } \\
\text { male } \%\end{array}$ & $\begin{array}{l}\text { Age inclusion } \\
\text { Median (IQR)/ } \\
\text { mean age (SD) }\end{array}$ & $\begin{array}{l}\text { Follow-up* } \\
\text { (days) }\end{array}$ & $\begin{array}{l}\text { Type } \\
\text { of } \\
\text { revisit }\end{array}$ & $\begin{array}{l}\text { Risk of bias } \\
\text { (high/low) }\end{array}$ \\
\hline Baker 2009 USA & $\mathrm{CP}$ & Article & Fever & Yes & $\begin{array}{l}280 \\
\text { NR }\end{array}$ & $\begin{array}{l}105 \\
\text { NR }\end{array}$ & $\begin{array}{l}3-36 \text { months } \\
\text { NR }\end{array}$ & $319-656 t$ & suR & Low \\
\hline Bloch 2013 USA & $\mathrm{RCT}$ & Article & All‡ & No & $\begin{array}{l}436 \\
54 \%\end{array}$ & $\begin{array}{l}216 \\
58 \%\end{array}$ & $\begin{array}{l}1 \text { month to } \\
18 \text { years } \\
\text { NR }\end{array}$ & $2-5$ & NA & Low \\
\hline $\begin{array}{l}\text { Browne } 2001 \\
\text { Australia }\end{array}$ & $\mathrm{BA}$ & Article & $\begin{array}{l}\text { GE, asthma, } \\
\text { croup }\end{array}$ & Yes & $\begin{array}{l}5534 \\
\text { NR }\end{array}$ & $\begin{array}{l}240 \\
\text { NR }\end{array}$ & NR & NR & suR & High \\
\hline $\begin{array}{l}\text { Considine } 2007 \\
\text { Australia }\end{array}$ & $\mathrm{BA}$ & Article & Fever & No & $\begin{array}{l}40 \\
N R\end{array}$ & $\begin{array}{l}15 \\
\text { NR }\end{array}$ & $\begin{array}{l}<16 \text { years } \\
3.1 \text { years } \pm 2.5 \\
\text { before } \\
1.8 \text { years } \pm 1.3 \text { after }\end{array}$ & 2 & NA & High \\
\hline Chande 1996 USA & $\mathrm{RCT}$ & Article & All & Yes & $\begin{array}{l}130 \\
59 \%\end{array}$ & $\begin{array}{l}37 \\
N R\end{array}$ & $\begin{array}{l}\text { All } \\
39 \text { months } ₫ \pm 36 \\
63 \text { months } \pm 58\end{array}$ & $\begin{array}{l}30,90 \text { and } \\
180\end{array}$ & suR & High \\
\hline Fagbuyi 2011 USA & $\mathrm{CP}$ & Article & Influenza-like & No & $\begin{array}{l}38646 \\
53 \%\end{array}$ & $\begin{array}{l}1091 \\
N R\end{array}$ & $\begin{array}{l}6 \text { months to } \\
21 \text { years } \\
82.3 \text { months } \pm 84.6\end{array}$ & 7 & $\mathrm{uR}$ & High \\
\hline Horne 1995 USA & $\mathrm{CP}$ & Article & All & No & $\begin{array}{l}250 \\
N R\end{array}$ & $\begin{array}{l}171 \\
\text { NR }\end{array}$ & $\begin{array}{l}\text { All } \\
\text { NR }\end{array}$ & 3 & NA & Low \\
\hline Ismail 2013 USA & $\mathrm{RCT}$ & Abstract & Fever & No & $\begin{array}{l}63 \\
16 \%\end{array}$ & NR & NR & 14 & NR & High \\
\hline Lawrence 2009 USA & $\mathrm{CR}$ & Article & All & Yes & $\begin{array}{l}40418 \\
N R\end{array}$ & $\begin{array}{l}979 \\
\text { NR }\end{array}$ & $\begin{array}{l}\text { NR } \\
2 \text { years }(0.5-7.0)\end{array}$ & 3 & suR & High \\
\hline Maguire $^{* *} 2011$ UK & $\mathrm{CP}$ & Article & Fever & No & $\begin{array}{l}220 \\
56 \%\end{array}$ & $\begin{array}{l}29 / 56 \\
\text { NR }\end{array}$ & $\begin{array}{l}<5 \text { years } \\
27 \% \leq 1 \text { years }\end{array}$ & NS & suR & High \\
\hline 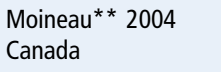 & $\mathrm{CR}$ & Abstract & GE & Yes & $\begin{array}{l}1862 \\
\text { NR }\end{array}$ & $\begin{array}{l}108 \\
\text { NR }\end{array}$ & $\begin{array}{l}\text { NR } \\
2.6 \text { years } \pm 2.8\end{array}$ & 7 & $\mathrm{uR}$ & High \\
\hline $\begin{array}{l}\text { O'Neill-Murphy } 2001 \\
\text { USA }\end{array}$ & BA & Article & Fever & No & $\begin{array}{l}87 \\
N R\end{array}$ & NR & $\begin{array}{l}3 \text { months to } 5 \text { years } \\
\text { NR }\end{array}$ & 14,56 & suR & High \\
\hline O'Neill 2001 USA & $\mathrm{CR}$ & Article & All & No & NR & NR & NR & NR & NA & High \\
\hline Patel 2009 USA & $\mathrm{nRCT}$ & Article & GE & No & $\begin{array}{l}291 \\
\text { NS }\end{array}$ & NA & $\begin{array}{l}3 \text { months to } \\
18 \text { years } \\
60 \%<1 \text { years }\end{array}$ & 1,2 & NA & High \\
\hline Porter 2000 USA & $\mathrm{CP}$ & Article & Fever & No & $\begin{array}{l}92 \\
N R\end{array}$ & NA & $\begin{array}{l}\leq 36 \text { months } \\
27,4 \text { years } \pm 9,2\end{array}$ & NA & NA & High \\
\hline Roland 2011 UK & $\mathrm{CP}$ & Abstract & Fever & No & $\begin{array}{l}457 \\
N R\end{array}$ & NR & NR & NR & $\mathrm{uR}$ & High \\
\hline Scarfone 1996 USA & $\mathrm{CP}$ & Article & All & No & $\begin{array}{l}179 \\
55 \%\end{array}$ & $\begin{array}{l}91 \\
N R\end{array}$ & $\begin{array}{l}\text { NR } \\
31 \text { monthstt }\end{array}$ & 1 & NA & Low \\
\hline Yang 2012 Taiwan & BA & Article & All & Yes & $\begin{array}{l}1285 \\
54 \%\end{array}$ & $\begin{array}{l}9 \\
56 \%\end{array}$ & $\begin{array}{l}\text { NR } \\
34 \text { months } \S \\
(0-207)\end{array}$ & 3 & suR & High \\
\hline
\end{tabular}

\footnotetext{
*Time until revisit.

tSee online supplementary information 2.

‡Common illnesses, without children with traumatic presenting symptoms.

$\S$ In the intervention group.

शIn the control group.

${ }^{* *}$ Studies included for both study aims (Maguire et al 2011 and Moineau et al 2004).

t+Mean $(\mathrm{Cl})$.

All, all ED diagnoses; BA, before after trial; $C P$, cohort study, prospective; $C R$, cohort study, retrospective; GE, gastroenteritis; NA, not applicable; NR, not recorded; $n R C T$,

non-randomised controlled trial; NS, not specified; RCT, randomised controlled trial; suR, scheduled and unscheduled revisit; uR, unscheduled revisit.
}

corresponding level of evidence as identified by the colours of the plus/minus signs, according to the best-evidence synthesis (details in online supplementary information $3 \mathrm{a}$ and $3 \mathrm{c}$ ).

\section{Moderate/limited evidence}

There was moderate evidence for the positive influence of a standardised follow-up programme (including, eg, a venue for handling calls after ED visits) ${ }^{8}$ on patient care and patient satisfaction. ${ }^{8} 9$ Limited evidence was found that clinical pathways at the ED resulted in a reduced admission rate, shortened length of stay and fewer revisits after discharge. ${ }^{10}$ We found limited evidence for risk factors associated with non-compliance of scheduled revisits; for example, parents' perception that their child is not severely ill, parents' age $(<21$ years) and ED physicians uncertainty about patients' return. ${ }^{11}$

Conflicting evidence

We found conflicting evidence for the association between safety-netting advice and the reduction of revisits. According to four studies, ${ }^{12-14}$ revisits could be reduced by providing consistent verbal and written discharge information regarding the natural history of disease ${ }^{13}$ and temperature measurement/ 
Table 2 Characteristics of included studies regarding the second study aim: determinants of revisits

\begin{tabular}{|c|c|c|c|c|c|c|c|c|c|c|}
\hline $\begin{array}{l}\text { Author } \\
\text { Year } \\
\text { Country }\end{array}$ & $\begin{array}{l}\text { Study } \\
\text { design }\end{array}$ & $\begin{array}{l}\text { Article/ } \\
\text { abstract }\end{array}$ & $\begin{array}{l}\text { Disease/ } \\
\text { working } \\
\text { diagnosis }\end{array}$ & $\begin{array}{l}\text { Primary } \\
\text { outcome: } \\
\text { revisits }\end{array}$ & $\begin{array}{l}\mathrm{N} \text { total, male } \\
\% \text { of total } \\
\text { population }\end{array}$ & $\begin{array}{l}\text { N outcome } \\
\text { (revisits), } \\
\text { male } \%\end{array}$ & $\begin{array}{l}\text { Age inclusion } \\
\text { Median (IQR)/ } \\
\text { mean age (SD) }\end{array}$ & $\begin{array}{l}\text { Follow-up* } \\
\text { (days) }\end{array}$ & $\begin{array}{l}\text { Type of } \\
\text { revisit }\end{array}$ & $\begin{array}{l}\text { Risk of } \\
\text { biast } \\
\text { (high/low) }\end{array}$ \\
\hline Alessandrini 2004 USA & CR & Article & All & Yes & $\begin{array}{l}54784 \\
\text { NR }\end{array}$ & $\begin{array}{l}1893 \\
N R\end{array}$ & $\begin{array}{l}\text { All } \\
4.6 \text { years } \pm 4.9 \ddagger\end{array}$ & 2 & suR & Low \\
\hline Ali 2012 USA & $\mathrm{CP}$ & Article & All & Yes & $\begin{array}{l}8742 \\
N R\end{array}$ & $\begin{array}{l}124 \\
52 \%\end{array}$ & $\begin{array}{l}\text { All } \\
3.0 \text { years }(1.1-12) \ddagger\end{array}$ & 3 & suR & High \\
\hline $\begin{array}{l}\text { Angoulvant } 2012 \\
\text { France }\end{array}$ & $\mathrm{CP}$ & Article & All§ & Yes & $\begin{array}{l}501 \\
N R\end{array}$ & $\begin{array}{l}206 \\
51 \%\end{array}$ & $\begin{array}{l}<6 \text { years } \\
18 \text { months }(7-39)\end{array}$ & 7 & suR & High \\
\hline Augustine 2013 USA & CS & Abstract & All & Yes & $\begin{array}{l}13 \\
N R\end{array}$ & $\begin{array}{l}13 \\
N R\end{array}$ & $\begin{array}{l}\text { All } \\
4.2 \text { years } \uparrow\end{array}$ & 2 & $\mathrm{uR}$ & High \\
\hline Berry 2013 USA & CR & Article & All & Yes & $\begin{array}{l}568845 \\
\text { NR }\end{array}$ & $\begin{array}{l}36734^{* *} \\
\text { NR }\end{array}$ & $\begin{array}{l}\leq 18 \text { years } \\
3 \text { years }(0-10)\end{array}$ & 30 & $\mathrm{uR}$ & Low \\
\hline Black 2010 UK & $\mathrm{CR}$ & Abstract & All & Yes & $\begin{array}{l}2345 \\
N R\end{array}$ & $\begin{array}{l}91 \\
\text { NR }\end{array}$ & $\begin{array}{l}<17 \text { years } \\
76 \%<5 \text { years }\end{array}$ & 3 & $\mathrm{uR}$ & High \\
\hline Callery 2010 UK & $\mathrm{CR}$ & Article & All & Yes & $\begin{array}{l}43372 \\
\text { NR }\end{array}$ & $\begin{array}{l}2433 \\
N R\end{array}$ & $\begin{array}{l}<15 \text { years } \\
\text { NR }\end{array}$ & 7 & suR & Low \\
\hline Chang 2008 Taiwan & $\mathrm{CR}$ & Article & All & No & $\begin{array}{l}3216 \\
58 \%\end{array}$ & $\begin{array}{l}188 \\
N R\end{array}$ & $\begin{array}{l}<18 \text { years } \\
5 \text { years } \pm 0.1\end{array}$ & 3 & suR & Low \\
\hline DePiero 2002 USA & CR & Article & All & Yes & $\begin{array}{l}51195 \\
\text { NR }\end{array}$ & $\begin{array}{l}261 \text { ** } \\
\text { NR }\end{array}$ & $\begin{array}{l}\text { All } \\
\text { NR }\end{array}$ & 3 & suR & Low \\
\hline Dunlop 2005 Australia & CR & Article & Fever & No & $26052 \%$ & $35 \mathrm{NR}$ & $\begin{array}{l}6 \text { months to } 6 \text { years } \\
25.7 \text { monthst } t\end{array}$ & 1 & suR & High \\
\hline Easter 2012 USA & CR & Article & All & Yes & $\begin{array}{l}97374 \\
\text { NR }\end{array}$ & $\begin{array}{l}1091^{* *} \\
52 \%\end{array}$ & $\begin{array}{l}0-21 \text { years } \\
52 \%<5 \text { years } \neq\end{array}$ & 4 & suR & Low \\
\hline Florin 2013 USA & CR & Article & Pneumonia & Yes & $\begin{array}{l}100615 \\
54 \%\end{array}$ & $\begin{array}{l}6439 \\
N R\end{array}$ & $\begin{array}{l}2 \text { months to } \\
18 \text { years } \\
3 \text { years }(1-6)\end{array}$ & 3 & suR & Low \\
\hline Freedman 2013 Canada & CR & Article & GE & Yes & $\begin{array}{l}3346 \\
55 \%\end{array}$ & $\begin{array}{l}526 \\
57 \%\end{array}$ & $\begin{array}{l}<18 \text { years } \\
3.4 \text { years } \pm 3.5\end{array}$ & 7 & $\mathrm{uR}$ & Low \\
\hline Gallagher 2013 USA & CR & Article & All & Yes & $\begin{array}{l}119792 \\
53 \%\end{array}$ & $\begin{array}{l}1499 * * \\
N R\end{array}$ & $\begin{array}{l}\text { All } \\
7.6 \text { years } \uparrow\end{array}$ & 3 & $\mathrm{uR}$ & Low \\
\hline Gaucher 2012 Canada & CR & Article & All & No & $\begin{array}{l}49146 \\
51 \%\end{array}$ & $\begin{array}{l}2534 \\
N R\end{array}$ & $\begin{array}{l}<19 \text { years } \\
62 \%<5 \text { years }\end{array}$ & 2 & $\mathrm{uR}$ & Low \\
\hline Goldman 2006 Canada & CR & Article & All & Yes & $\begin{array}{l}37725 \\
\text { NR }\end{array}$ & $\begin{array}{l}1990 \\
N R\end{array}$ & $\begin{array}{l}<19 \text { years } \\
18 \%<1 \text { year }\end{array}$ & 3 & $\mathrm{uR}$ & Low \\
\hline Goldman 2011 Canada & CR & Article & All & Yes & $\begin{array}{l}2062 \\
55 \%\end{array}$ & $\begin{array}{l}353^{* *} \\
59 \%\end{array}$ & $\begin{array}{l}<19 \text { years } \\
57 \text { months }(0-215)\end{array}$ & 3 & suR & High \\
\hline Gregor 2009 USA & $\mathrm{CP}$ & Article & RTI/GE & No & $\begin{array}{l}455 \\
59 \%\end{array}$ & $\begin{array}{l}49 \\
N R\end{array}$ & $\begin{array}{l}6 \text { weeks to } 8 \text { years } \\
1.9 \text { years } \pm 1.9\end{array}$ & 60 & suR & High \\
\hline Hacking 2012 UK & CR & Abstract & All & Yes & $\begin{array}{l}2453 \\
\text { NR }\end{array}$ & $\begin{array}{l}130 \\
N R\end{array}$ & $\begin{array}{l}\text { NR } \\
4 \text { yearstt }\end{array}$ & NR & $\mathrm{uR}$ & High \\
\hline Jacobstein 2005 USA & $\mathrm{CC}$ & Article & Fever & Yes & $\begin{array}{l}15384 \\
54 \%\end{array}$ & $\begin{array}{l}165 \\
54 \%\end{array}$ & $\begin{array}{l}\text { All } \\
38 \text { months } \pm 43\end{array}$ & 3 & $\mathrm{uR}$ & Low \\
\hline Jain 2010 USA & CR & Article & All & No & $\begin{array}{l}452868 \\
54 \%\end{array}$ & $\begin{array}{l}17335 \\
N R\end{array}$ & $\begin{array}{l}<19 \text { years } \\
22 \%<1 \text { years }\end{array}$ & 3 & suR & Low \\
\hline $\begin{array}{l}\text { Klein-Kremer } 2011 \\
\text { Canada }\end{array}$ & CR & Article & Fever & Yes & $\begin{array}{l}397 \\
\text { NR }\end{array}$ & $\begin{array}{l}92 \\
67 \%\end{array}$ & $\begin{array}{l}3-36 \text { months } \\
17 \text { months } \pm 8 \ddagger\end{array}$ & 3 & suR & High \\
\hline Lal et al 1999 UK & $\mathrm{CP}$ & Article & All & Yes & $\begin{array}{l}7328 \\
\text { NR }\end{array}$ & $\begin{array}{l}65 \\
N R\end{array}$ & NR & 3 & $\mathrm{uR}$ & High \\
\hline LeDuc 2006 USA & $\mathrm{CP}$ & Article & All & Yes & $\begin{array}{l}932 \\
\text { NR }\end{array}$ & $\begin{array}{l}237 \\
49 \%\end{array}$ & $\begin{array}{l}\text { All } \\
4 \text { years }\end{array}$ & 2,90 & suR & High \\
\hline Liberman 2012 USA & CR & Article & RTI & No & $\begin{array}{l}467 \\
59 \%\end{array}$ & $\begin{array}{l}189 \\
N R\end{array}$ & $\begin{array}{l}<19 \text { years } \\
\text { NR }\end{array}$ & 7,30 & suR & Low \\
\hline Logue 2013 Canada & $C R$ & Article & All & Yes & $\begin{array}{l}1173 \\
N R\end{array}$ & $\begin{array}{l}261 \\
61 \%\end{array}$ & $\begin{array}{l}\text { All } \\
4.4 \text { years } 9\end{array}$ & 3 & suR & High \\
\hline Maguireł‡ 2011 UK & $\mathrm{CP}$ & Article & Fever & No & $\begin{array}{l}220 \\
56 \%\end{array}$ & $\begin{array}{l}127 \\
N R\end{array}$ & $\begin{array}{l}<5 \text { years } \\
27 \% \leq 1 \text { years }\end{array}$ & NS & suR & High \\
\hline Mansbach 2008 USA & $\mathrm{CP}$ & Article & Bronchiolitis & No & $\begin{array}{l}1456 \\
58 \%\end{array}$ & $\begin{array}{l}837 \\
58 \%\end{array}$ & $\begin{array}{l}<2 \text { years } \\
6.9(4.2-11.3) \S \S\end{array}$ & 14 & NS & Low \\
\hline Michelson 2012 USA & $\mathrm{CR}$ & Article & All & No & $\begin{array}{l}198778 \\
\text { NR }\end{array}$ & $\begin{array}{l}7281 \\
N R\end{array}$ & $\begin{array}{l}\text { All } \\
10 \%<1 \text { years }\end{array}$ & 2 & suR & High \\
\hline Mintegui 2000 Spain & CR & Abstract & All & Yes & 3667 NR & $495 \mathrm{NR}$ & All NR & 7 & $\mathrm{uR}$ & High \\
\hline Mistry 2007 USA & $\mathrm{CP}$ & Article & Fever & Yes & $\begin{array}{l}322 \\
57 \%\end{array}$ & $\begin{array}{l}76 \\
\text { NR }\end{array}$ & $\begin{array}{l}28 \text { days to } 17 \text { years } \\
31.5 \text { months } 9\end{array}$ & 10 & $\mathrm{uR}$ & High \\
\hline
\end{tabular}


Table 2 Continued

\begin{tabular}{|c|c|c|c|c|c|c|c|c|c|c|}
\hline $\begin{array}{l}\text { Author } \\
\text { Year } \\
\text { Country }\end{array}$ & $\begin{array}{l}\text { Study } \\
\text { design }\end{array}$ & $\begin{array}{l}\text { Article/ } \\
\text { abstract }\end{array}$ & $\begin{array}{l}\text { Disease/ } \\
\text { working } \\
\text { diagnosis }\end{array}$ & $\begin{array}{l}\text { Primary } \\
\text { outcome: } \\
\text { revisits }\end{array}$ & $\begin{array}{l}\mathrm{N} \text { total, male } \\
\% \text { of total } \\
\text { population }\end{array}$ & $\begin{array}{l}\text { N outcome } \\
\text { (revisits), } \\
\text { male } \%\end{array}$ & $\begin{array}{l}\text { Age inclusion } \\
\text { Median (IQR)/ } \\
\text { mean age (SD) }\end{array}$ & $\begin{array}{l}\text { Follow-up* } \\
\text { (days) }\end{array}$ & $\begin{array}{l}\text { Type of } \\
\text { revisit }\end{array}$ & $\begin{array}{l}\text { Risk of } \\
\text { biast } \\
\text { (high/low) }\end{array}$ \\
\hline Mistry 2009 USA & $\mathrm{CP}$ & Article & Fever & No & $\begin{array}{l}97 \\
56 \%\end{array}$ & $\begin{array}{l}18 \\
N R\end{array}$ & $\begin{array}{l}2-18 \text { years } \\
58.7 \text { months } \pm 40.1\end{array}$ & $7-10$ & $\mathrm{uR}$ & High \\
\hline $\begin{array}{l}\text { Moineau } \ddagger 2004 \\
\text { Canada }\end{array}$ & $\mathrm{CR}$ & Abstract & GE & Yes & $\begin{array}{l}1862 \\
N R\end{array}$ & $\begin{array}{l}108 \\
N R\end{array}$ & $\begin{array}{l}\text { NR } \\
2.6 \text { years } \pm 2.8\end{array}$ & 7 & $\mathrm{uR}$ & High \\
\hline O'Loughlin 2012 UK & $\mathrm{CR}$ & Article & All & Yes & $\begin{array}{l}10573 \\
N R\end{array}$ & $\begin{array}{l}532 \\
\text { NR }\end{array}$ & $\begin{array}{l}<16 \text { years } \\
34 \%<2 \text { years }\end{array}$ & 7 & $\mathrm{uR}$ & High \\
\hline Roback 1997 USA & CC & Article & Bronchiolitis & Yes & $\begin{array}{l}181 \\
N R\end{array}$ & $\begin{array}{l}57 \\
N R\end{array}$ & $\begin{array}{l}<1 \text { year } \\
\text { NR }\end{array}$ & 4 & NS & High \\
\hline Roggen 2012 Belgium & $\mathrm{CR}$ & Abstract & All§ & Yes & $\begin{array}{l}46386 \\
N R\end{array}$ & $\begin{array}{l}1864 \\
N R\end{array}$ & $\begin{array}{l}<16 \text { years } \\
\text { NR }\end{array}$ & 3 & suR & High \\
\hline $\begin{array}{l}\text { Samuels-Kalow } 2013 \\
\text { Canada }\end{array}$ & $\mathrm{CR}$ & Abstract & Fever & Yes & $\begin{array}{l}202 \\
\text { NR }\end{array}$ & $\begin{array}{l}14 \\
N R\end{array}$ & $\begin{array}{l}\text { 2-24 months } \\
\text { NR }\end{array}$ & 3 & suR & High \\
\hline Sartain 2002 UK & $\mathrm{RCT}$ & Article & All & No & 399 & $\begin{array}{l}31 \\
\text { NS }\end{array}$ & $\begin{array}{l}\text { All } \\
25.7 \text { months }\end{array}$ & 90 & suR & High \\
\hline Seow 2007 Taiwan & $\mathrm{CR}$ & Article & Fever & No & $\begin{array}{l}345 \\
47 \%\end{array}$ & $\begin{array}{l}115 \\
N R\end{array}$ & $\begin{array}{l}\text { 3-36 months } \\
\text { NR }\end{array}$ & 3 & $\mathrm{uR}$ & Low \\
\hline Simmons 2012 UK & $\mathrm{CR}$ & Abstract & All & Yes & NR & $\begin{array}{l}51 \\
N R\end{array}$ & $\begin{array}{l}\text { All } \\
59 \%<2 \text { years }\end{array}$ & 7 & $\mathrm{uR}$ & High \\
\hline Small 2005 UK & $\mathrm{CP}$ & Article & GE & No & $\begin{array}{l}112 \\
\text { NR }\end{array}$ & $\begin{array}{l}56 \\
\text { NR }\end{array}$ & $\begin{array}{l}1-6 \text { years } \\
1.9(1.3)^{* *}\end{array}$ & 7,30 & suR & Low \\
\hline Zimmerman 1996 USA & $\mathrm{CR}$ & Article & All & Yes & $\begin{array}{l}5228 \\
58 \%\end{array}$ & $\begin{array}{l}242 \\
N R\end{array}$ & $\begin{array}{l}<18 \text { years } \\
13 \%<1 \text { years }\end{array}$ & 14 & suR & Low \\
\hline
\end{tabular}

*Time until revisit.

tMinimum and maximum.

$\ddagger$ Of the number of children with revisits.

$\S$ Common illnesses, without children with traumatic complaints.

१IMean (CI).

${ }^{* *}$ Revisits requiring admission.

t†Median (IQR).

$\ddagger \ddagger$ Studies included for both study aims (Maguire et $a l^{12}$ and Moineau et $a l^{13}$ ).

$\S \S 0 f$ the number of children sent home.

All, all emergency department diagnoses; CC, case-control study; CP, cohort study, prospective; $C R$, cohort study, retrospective; CS, cross-sectional study; GE, gastroenteritis; NR, not recorded; RCT, randomised controlled trial; RTI, respiratory tract illnesses; suR, scheduled and unscheduled revisit; uR, unscheduled revisit.

treatment. ${ }^{14}$ In contrast, other studies concluded that the provision of safety-net advice did not affect the number of revisits. $^{15} 16$ We found conflicting evidence for the association between educational interventions at the ED and parental recall of discharge instructions or revisits. ${ }^{16-24}$ One study reported that video home management of fever improved caregiver's knowledge of fever, but did not decrease ED use. ${ }^{18}$ There was conflicting evidence about the role of telephone follow-up as safety-netting strategy. One study reported that this was an effective way of providing, for example, health information, managing remaining symptoms and recognising complications. ${ }^{25}$ In contrast, another study advocated caution in the implementation of telephone follow-up because of moderate success rate in reaching patients. $^{26}$

\section{Determinants of revisits}

Figure 3 presents an overview of all determinants of revisits described in the included studies $(n=42)$, their association with revisits and the corresponding level of evidence, according to the best-evidence synthesis (details in online supplementary information $3 b$ and $3 c$ ).

\section{Strong evidence}

Child characteristics

We found strong evidence for the association of ED revisits with younger children, ranging from $\leq 12$ months until $<6$ years. $^{12}$ 27-40 Moreover, for the association between medical history and revisits, although including heterogeneous definitions, we found strong evidence. ${ }^{12} 2835374142$ Maquire et al ${ }^{12}$ concluded that history of illness in febrile children was one of the reasons for parental advice-seeking behaviour. However, for children with bronchiolitis, this association was conflicting. ${ }^{35} 41$ With strong evidence, no association was found between gender and revisits to the $\mathrm{ED}^{27} 3043$ or revisits to the primary care provider. $^{33}$ Gender was neither discriminating in the comparison of admitted children with the discharged ones after revisiting the ED nor a prognostic factor in safe discharge of children with bronchiolitis. $^{354144}$

\section{Social and demographic characteristics}

There was conflicting evidence that ED revisits were associated with ED crowding. ${ }^{27} 2942$ Two studies were positively associated with revisits, ${ }^{39} 45$ and three other studies were even associated with lower ED crowding during late evening or night shifts. $^{32} 4046$

\section{Disease characteristics}

Strong evidence was found for the association of revisits of children with symptoms of infectious diseases ${ }^{9} 29313335-3739434547-50$ or respiratory symptoms $29303537414547-4951$ compared with all ED revisits. Strong evidence was found for the association between revisits and seizures or other nervous system diseases. ${ }^{27} 3739$ Lastly, strong association was found between progression/persistence of symptoms and revisits. ${ }^{9} 13363839444851-56$ 


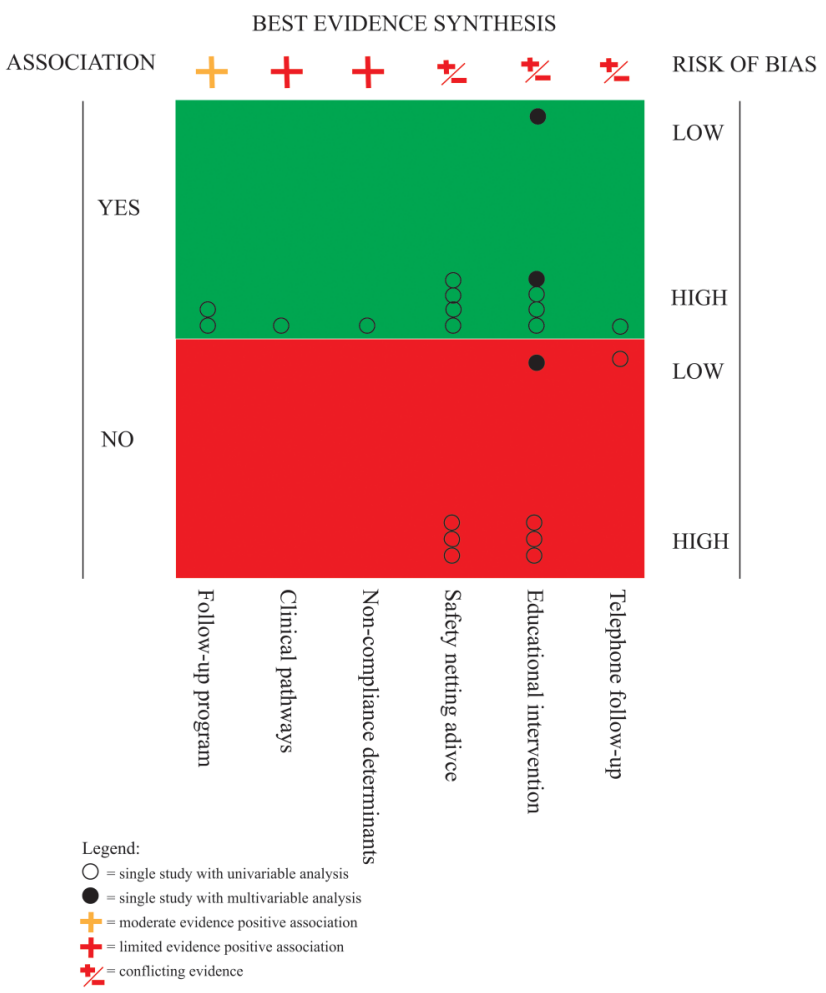

Figure 2 Level of evidence safety-netting strategies according to the best-evidence synthesis.

\section{Physician characteristics}

We found no association between physicians' characteristics, such as being paediatrician or resident ${ }^{42} 57$ or physicians' years of experience, ${ }^{4158}$ and revisits. ${ }^{41425758}$

Process characteristics

We observed strong evidence for the absence of the association between revisits and the performance of diagnostic tests or therapeutic interventions at the index visit. ${ }^{43} 48555960$

\section{Limited/moderate evidence}

\section{Child characteristics}

Mistry et al studied a health-related quality-of-life instrument (PedsQL). There was limited evidence for the association between lower changes in PedsQL scores and ED revisits, which implied less improved quality of life for the revisiting child. ${ }^{61}$

\section{Process characteristics}

No association was found between revisits and paediatric hospital at home service compared with conventional hospital care for children suffering from breathing difficulty, diarrhoea and vomiting, or fever. ${ }^{62}$ We found no association between revisits and children with acute gastroenteritis admitted to hospital compared with a comparable group of children managed at home. ${ }^{63}$

Conflicting evidence

Child characteristics

There was conflicting evidence for the association between ethnicity and revisits. In disease-specific studies (bronchiolitis and

\section{BEST EVIDENCE SYNTHESIS}

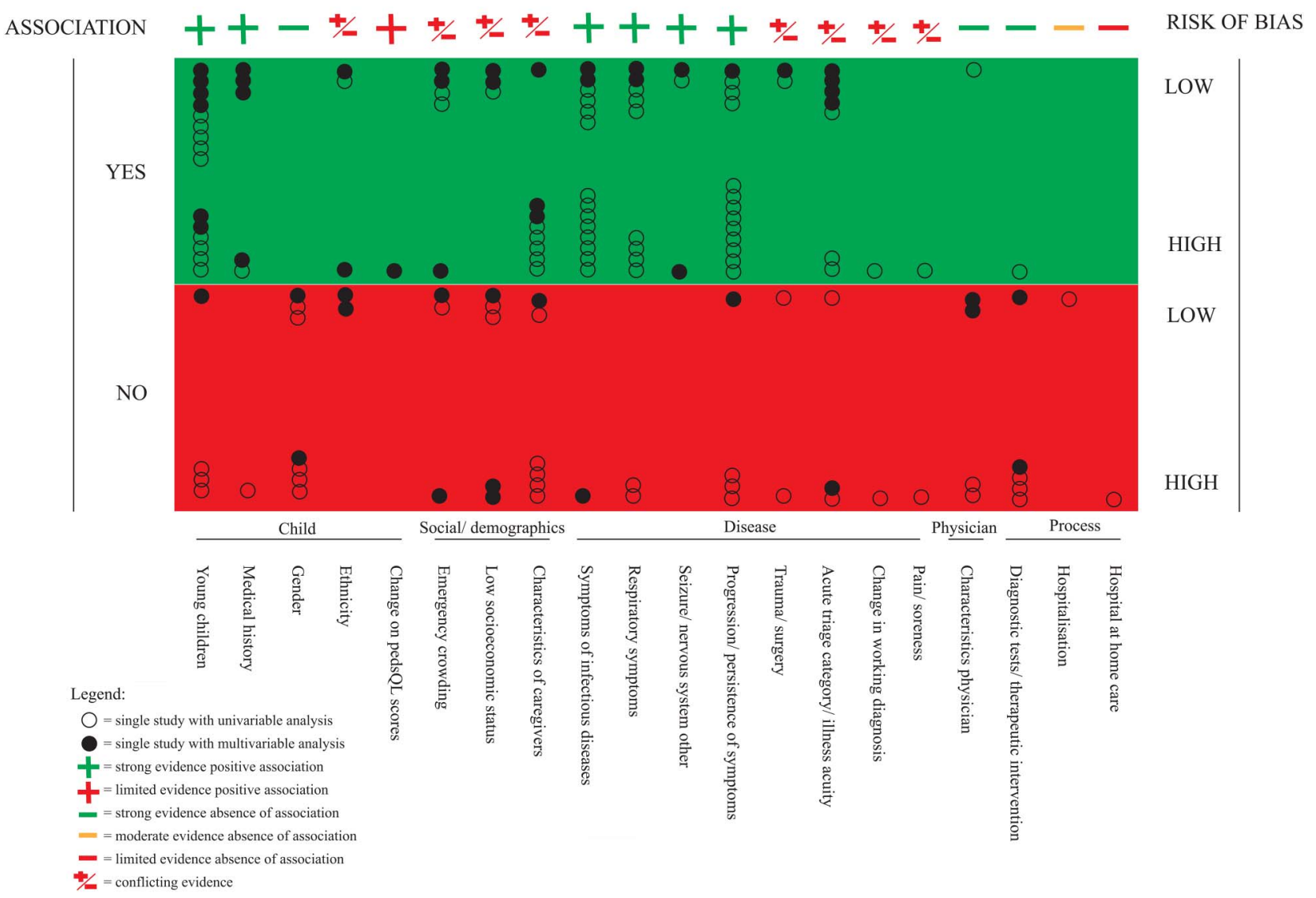

Figure 3 Level of evidence determinants of revisits according to the best-evidence synthesis. 
fever), ethnicity was not associated with revisits ${ }^{42} 35$ in contrast to studies including the total ED population. ${ }^{27} 3037$

\section{Social and demographic characteristics}

There was conflicting evidence for the association between revisits and characteristics of caregivers. For example, caregiver's age, marital status and presence/age of other children were not associated with revisits in five studies. ${ }^{33} 42-4455$ In contrast, other studies concluded that language spoken at home or single caregivers were associated with revisits. ${ }^{9} \quad 12 \quad 28 \quad 36 \quad 40 \quad 50 \quad 51 \quad 64$ Next, we found conflicting evidence for the association between lower socioeconomic status and revisits. ${ }^{27} 28303337404245$

\section{Disease characteristics}

Associations between trauma, surgical problems or pain ${ }^{43} 48$ and revisits were conflicting. ${ }^{29} 303748$ Conflicting evidence was found for the association between revisits in change of working diagnosis $^{4447}$ and ED triage acuity. ${ }^{132829323336384042}$

\section{DISCUSSION}

Follow-up after discharge and determinants of revisits: main outcomes

Limited evidence was observed for different strategies of safety netting, with educational interventions being mostly studied. Identified determinants of children at risk for revisits included young children, relevant medical history, infectious/respiratory symptoms or seizures and progression/persistence of symptoms. No association with revisits was found for gender, emergency crowding, physicians' characteristics and diagnostic tests and/or therapeutic interventions at the index ED. For other described determinants, no statement was possible due to conflicting evidence.

\section{Strengths and weaknesses of this review}

The development of evidence-based strategies of safety netting is a challenging new topic. Available studies describing revisits of the ED population and their characteristics vary in populations, study aims and methodology. The main strength of this systematic review is combining all information on determinants of revisits using a best-evidence synthesis. Most studies about safety netting are rather descriptive, and did not study their effectiveness. ${ }^{15}$ In our review, we summarised the literature that evaluated the clinical consequences of their safety-netting intervention.

This review has some limitations. Because of the heterogeneity of the studies, we could not perform a meta-analysis. This systematic review is limited to the provision of whether there is evidence for a significant association or not. This approach limits the interpretation and clinical relevance of the reported associations, but is a consequence of the large heterogeneity of present studies on this topic. Second, there is no standardised risk-of-bias assessment method for the variation of study designs and outcomes included in this systematic review. To overcome lack of general accepted thresholds determining the study's risk of bias, and to include relevance to the research question on the risk-of-bias criteria, ${ }^{65}$ we used the MINORS risk-of-bias criteria. ${ }^{6}$ We added two important items, which would be the most appropriate for our included studies. With this approach, we aimed to perform best available systematic risk-of-bias analysis. We classified determinants to 'strong evidence' on the presence of low risk-of-bias studies, although high risk-of-bias studies may also have studied the same determinants (see online supplementary information 2).
Furthermore, there are limitations embedded in the study design of the included studies itself. The majority of studies are analysed with univariable statistical approaches, with only 35\% (20/58) of the studies using multivariable statistical analysis. It remains unknown to what extent the determinants are independently associated with revisits. Second, although we followed the focus of most studies by defining 'revisits' as proxy for high-risk populations of failed safety-netting strategies, hospitalisation after revisiting the ED is probably the most effective outcome to evaluate this topic. However, study of this outcome is limited due to its low prevalence. Third, some study characteristics increased heterogeneity between our different determinant categories. For example, determinants were not always specified, for example, 'history of illnesses' was not further described in the study of Maguire et al. Furthermore, outcome measures were not homogenous and included, for example, revisits or admission after revisit. Finally, study comparisons varied between revisits versus total ED population or subgroups of revisits (discharged vs admitted children).

\section{Implications for clinical practice and future research}

A content of safety-net advice, as included in the National Institute for Health and Care Excellence (NICE) guideline, ${ }^{66}$ has been published in relation to general practice where consensus was reached among general practitioners and paediatric ED consultants using a modified Delphi approach. ${ }^{1}{ }^{4}$ Safety-netting advice should include: (1) the existence of uncertainty, (2) what exactly to look out for, (3) how exactly to seek further help, (4) what to expect about time course. Our systematic review shows that a variety of safety-netting techniques are used, but the effective components or the best way to perform remains unknown, as has been identified by others. ${ }^{15}$ Second, we generated answers on what determinants are associated with revisits, and those who are not. Moreover, the conclusions of our review can improve homogeneity in study design on follow-up strategies, and can add to progress in this research area. In essence, the importance of this knowledge should be combined with parent-related factors as their ability to understand and to comply with the designed safetynetting strategy. ${ }^{11}$ Lastly, one notable gap in safety-netting literature is its time frame strategy. The NICE fever guideline claims 'to arrange a follow-up appointment at a certain time and place'. ${ }^{67} \mathrm{In}$ future research, we need to study the (efficacy of) safety-netting strategies in which the aspect of time is taken into account. ${ }^{567}$

\section{CONCLUSION}

Determination of a high-risk group in need for safety-netting strategies in paediatric emergency care remains difficult. We identified a set of strongly associated determinants of revisits that could be used for this identification; being young children, relevant medical history, infectious/respiratory symptoms or seizures and progression/persistence of symptoms. Gaps remain on intervention studies concerning specific application of a uniform safety-netting strategy and its included time frame.

Acknowledgements We gratefully acknowledge Wichor Bramer, medical librarian at the Erasmus University Rotterdam, for assisting us with the systematic literature search for this systematic review.

Contributors EdV-K substantially contributed to the conception and design of the study. She participated in the literature search and assessed studies for eligibility. She undertook data extraction, risk-of-bias assessment and performed the best-evidence synthesis. She drafted the initial manuscript, and approved the final manuscript as submitted. DHFG substantially contributed to the conception and design of the study, and reviewed and revised the manuscript. She participated in the risk-of-bias assessment, and approved the final manuscript as submitted. MW participated in the literature search, and assessed studies for eligibility. She reviewed and revised the manuscript, and approved the final manuscript as submitted. HAM substantially contributed to the conception and design of the study. She reviewed 
and revised the manuscript. She participated in discussion about each step of the results, and approved the final manuscript as submitted. RO substantially contributed to the conception and design of the study. She reviewed and revised the manuscript, and approved the risk-of-bias assessment and best-evidence synthesis analyses. She participated and supervised the discussion about each step of the results, and approved the final manuscript as submitted.

Funding EdV-K is supported by ZonMW, a Dutch organisation for health research and development. The study sponsor had no role in study design; in the collection, analysis and interpretation of data; in the writing of the report or in the decision to submit the paper for publication.

Competing interests None declared.

Provenance and peer review Not commissioned; externally peer reviewed.

\section{REFERENCES}

1 Almond S, Mant D, Thompson M. Diagnostic safety-netting. Br J Gen Pract 2009;59:872-4; discussion 4 Online.

2 Neighbour R. The inner consultation. Oxford: Radcliffe Publishing, 2004.

3 Roland D, Jones C, Neill S, et al. Safety netting in healthcare settings: what it means, and for whom? Arch Dis Child Educ Pract Ed 2014;99:48-53.

4 Van den Bruel A, Thompson M, Buntinx F, et al. Clinicians' gut feeling about serious infections in children: observational study. BMJ 2012;345:e6144

5 Jones $\mathrm{CH}$, Neill $\mathrm{S}$, Lakhanpaul $\mathrm{M}$, et al. The safety netting behaviour of first contact clinicians: a qualitative study. BMC Fam Pract 2013;14:140.

6 Slim K, Nini E, Forestier D, et al. Methodological index for non-randomized studies (minors): development and validation of a new instrument. ANZ J Surg 2003;73:712-16

7 van Tulder M, Furlan A, Bombardier C, et al. Editorial Board of the Cochrane Collaboration Back Review G. Updated method guidelines for systematic reviews in the Cochrane Collaboration back review group. Spine (Phila Pa 1976) 2003;28:1290-9.

8 O'Neill K, Silvestri A, McDaniel-Yakscoe N. A pediatric emergency department follow-up system: completing the cycle of care. Pediatr Emerg Care 2001;17:392-5.

9 Augustine EM, Kreling BA, Chamberlain JM. The parent perspective on return emergency department visits. J Invest Med 2013;61:678.

10 Browne GJ, Giles $\mathrm{H}, \mathrm{McC}$ askill ME, et al. The benefits of using clinical pathways for managing acute paediatric illness in an emergency department. J Qual Clin Pract 2001:21:50-5.

11 Scarfone RJ, Joffe MD, Wiley JF, et al. Noncompliance with scheduled revisits to a pediatric emergency department. Arch Pediatr Adolesc Med 1996;150:948-53.

12 Maguire S, Ranmal R, Komulainen $S$, et al. Which urgent care services do febrile children use and why? Arch Dis Child 2011;96:810-16.

13 Moineau G, McKinnon A, Gaboury I, et al. Unscheduled return visits for gastroenteritis to a pediatric emergency department. Pediatric Research 2004;55:129A

14 Porter RS, Wenger FG. Diagnosis and treatment of pediatric fever by caretakers. J Emerg Med 2000;19:1-4.

15 Roland D, Geliot T, Patel A. Delivering safety net advice and the emergency department clinical quality indicator of unplanned re-attendance in children. Emerg Med J 2011;28:A13.

16 Fagbuyi DB, Brown KM, Mathison DJ, et al. A rapid medical screening process improves emergency department patient flow during surge associated with novel H1N1 influenza virus. Ann Emerg Med 2011;57:52-9.

17 Patel B, Kennebeck SS, Caviness AC, et al. Use of a discharge facilitator improves recall of emergency department discharge instructions for acute gastroenteritis. Pediatr Emerg Care 2009;25:558-64.

18 Baker MD, Monroe KW, King WD, et al. Effectiveness of fever education in a pediatric emergency department. Pediatr Emerg Care 2009;25:565-8.

19 Considine J, Brennan D. Effect of an evidence-based education programme on ED discharge advice for febrile children. J Clin Nurs 2007;16:1687-94.

20 Ismail S, McIntosh M, Kalynych C, et al. Impact of video discharge instructions from the emergency department in regard to caregiver understanding of their child's fever and closed head injury. Ann Emerg Med 2013;62:S17.

21 Bloch SA, Bloch AJ. Using video discharge instructions as an adjunct to standard written instructions improved caregivers' understanding of their child's emergency department visit, plan, and follow-up: A randomized controlled trial. Pediatr Emerg Care 2013;29:699-704.

22 O'Neill-Murphy K, Liebman M, Barnsteiner JH. Fever education: does it reduce parent fever anxiety? Pediatr Emerg Care 2001;17:47-51.

23 Chande VT, Wyss N, Exum V. Educational interventions to alter pediatric emergency department utilization patterns. Arch Pediatr Adolesc Med 1996;150:525-8

24 Lawrence LM, Jenkins CA, Zhou C, et al. The effect of diagnosis-specific computerized discharge instructions on 72-hour return visits to the pediatric emergency department. Pediatr Emerg Care 2009;25:733-8,
25 Yang C, Chen CM. Effects of post-discharge telephone calls on the rate of emergency department visits in paediatric patients. J Paediatr Child Health 2012;48:931-5.

26 Horne A, Ros SP. Telephone follow-up of patients discharged from the emergency department: how reliable? Pediatr Emerg Care 1995;11:173-5.

27 LeDuc K, Rosebrook H, Rannie M, et al. Pediatric emergency department recidivism: Demographic characteristics and diagnostic predictors. J Emerg Nurs 2006:32:131-8.

28 Gregor MA, Wheeler JRC, Stanley RM, et al. Caregiver adherence to follow-up after an emergency department visit for common pediatric illnesses: Impact on future ED use. Med Care 2009;47:326-33.

29 Alessandrini EA, Lavelle JM, Grenfell SM, et al. Return visits to a pediatric emergency department. Pediatr Emerg Care 2004;20:166-71.

30 Zimmerman DR, McCarten-Gibbs KA, DeNoble DH, et al. Repeat pediatric visits to a general emergency department. Ann Emerg Med 1996;28:467-73.

31 Black L. Unscheduled re-attendances to a paediatric emergency department: an audit. Emerg Med J 2010;27:A9-10.

32 Goldman RD, Ong M, Macpherson A. Unscheduled return visits to the pediatric emergency department-one-year experience. Pediatr Emerg Care 2006;22:545-9.

33 Liberman DB, Shelef DQ, He J, et al. Low rates of follow-up with primary care providers after pediatric emergency department visits for respiratory tract IIInesses. Pediatr Emerg Care 2012;28:956-61.

34 O'Loughlin K, Hacking KA, Simmons N, et al. Paediatric unplanned reattendance rate: A\&E clinical quality indicators. Arch Dis Child 2013;98:211-13.

35 Mansbach JM, Clark S, Christopher NC, et al. Prospective multicenter study of bronchiolitis: predicting safe discharges from the emergency department. Pediatrics 2008;121:680-8.

36 Logue EP, Ali S, Spiers J, et al. Characteristics of patients and families who make early return visits to the pediatric emergency department. Open Access Emerg Med 2013;5:9-15

37 Berry JG, Toomey SL, Zaslavsky AM, et al. Pediatric readmission prevalence and variability across hospitals. JAMA 2013;309:372-80.

38 Freedman SB, Thull-Freedman JD, Rumantir M, et al. Emergency department revisits in children with gastroenteritis. J Pediatr Gastroenterol Nutr 2013;57:612-18.

39 Easter JS, Bachur R. Physicians' assessment of pediatric returns to the emergency department. J Emerg Med 2013;44:682-8.

40 Gallagher RA, Porter S, Monuteaux MC, et al. Unscheduled return visits to the emergency department: the impact of language. Pediatr Emerg Care 2013:29:579-83.

41 Roback MG, Baskin MN. Failure of oxygen saturation and clinical assessment to predict which patients with bronchiolitis discharged from the emergency department will return requiring admission. Pediatr Emerg Care 1997;13:9-11.

42 Jacobstein CR, Alessandrini EA, Lavelle JM, et al. Unscheduled revisits to a pediatric emergency department: risk factors for children with fever or infection-related complaints. Pediatr Emerg Care 2005;21:816-21.

43 Angoulvant $F$, Jumel $S$, Prot-Labarthe $S$, et al. Multiple health care visits related to a pediatric emergency visit for young children with common illnesses. Eur J Pediatr 2013:172:797-802.

44 Goldman RD, Kapoor A, Mehta S. Children admitted to the hospital after returning to the emergency department within 72 hours. Pediatr Emerg Care 2011;27:808-11.

45 Callery P, Kyle RG, Campbell M, et al. Readmission in children's emergency care: An analysis of hospital episode statistics. Arch Dis Child 2010;95:341-6.

46 Michelson KA, Monuteaux MC, Stack AM, et al. Pediatric emergency department crowding is associated with a lower likelihood of hospital admission. Acad Emerg Med 2012;19:816-20.

47 Mintegui Raso S, Benito Fernandez J, Vazquez Ronco MA, et al. Children's unscheduled return visits to an emergency department. An Esp Pediatr 2000;52:542-7.

48 Klein-Kremer A, Goldman RD. Return visits to the emergency department among febrile children 3 to 36 months of age. Pediatr Emerg Care 2011:27:1126-9.

49 Hacking K, Christian W. Clinical quality indicators in the children's emergency department-why do children re-attend? Arch Dis Child 2012;97:A144-A5.

50 Simmons N, Syahanee R, Marzouk O, et al. Audit of unplanned re-attendance to a paediatric emergency department. Arch Dis Child 2012;97:A153.

51 Lal MK, Kibirige MS. Unscheduled return visits within 72 hours to an assessment unit. Arch Dis Child 1999:80:455-8.

52 Ali $A B$, Place $R$, Howell J, et al. Early pediatric emergency department return visits: a prospective patient-centric assessment. Clin Pediatr 2012;51:651-8.

53 Depiero AD, Ochsenschlager DW, Chamberlain JM. Analysis of pediatric hospitalizations after emergency department release as a quality improvement tool. Ann Emerg Med 2002;39:159-63.

54 Dunlop S, Taitz J. Retrospective review of the management of simple febrile convulsions at a tertiary paediatric institution. J Paediatr Child Health 2005;41:647-51

55 Mistry RD, Stevens MW, Gorelick MH. Short-term outcomes of pediatric emergency department febrile illnesses. Pediatr Emerg Care 2007;23:617-23. 
56 Roggen I, Van Berlaer G, Lauwaert D, et al. Hospitalization rate and diagnosis severity increase on children's return visits to the ED. Acad Emerg Med 2012;19:773.

57 Chang YC, Lo HC, Tzeng YM, et al. Comparative clinical practice of residents and attending physicians who care for pediatric patients in the emergency department. Pediatr Emerg Care 2008;24:364-9.

58 Gaucher N, Bailey B, Gravel J. Impact of physicians' characteristics on the admission risk among children visiting a pediatric emergency department. Pediatr Emerg Care 2012;28:120-4.

59 Jain S, Elon LK, Johnson BA, et al. Physician practice variation in the pediatric emergency department and its impact on resource use and quality of care. Pediatr Emerg Care 2010;26:902-8.

60 Florin TA, French B, Zorc JJ, et al. Variation in emergency department diagnostic testing and disposition outcomes in pneumonia. Pediatrics 2013;132:237-44.

61 Mistry RD, Stevens MW, Gorelick MH. Health-related quality of life for pediatric emergency department febrile illnesses: an evaluation of the Pediatric Quality of Life Inventory(trademark) 4.0 generic core scales. Health Qual Life Outcomes 2009;7:5.
62 Sartain SA, Maxwell MJ, Todd PJ, et al. Randomised controlled trial comparing an acute paediatric hospital at home scheme with conventional hospital care. Arch Dis Child 2002;87:371-5.

63 Small F, Alderdice F, McCusker C, et al. A prospective cohort study comparing hospital admission for gastro-enteritis with home management. Child Care Health Dev 2005;31:555-62.

64 Samuels-Kalow ME, Stack AM, Amico K, et al. The association between parental language and 72-hour revisits following pediatric emergency department discharge. Acad Emerg Med 2013;20:S188.

65 Hayden JA, Cote $\mathrm{P}$, Bombardier C. Evaluation of the quality of prognosis studies in systematic reviews. Ann Intern Med 2006;144:427-37.

66 Fields $\mathrm{E}$, Chard J, Murphy MS, et al. Assessment and initial management of feverish illness in children younger than 5 years: summary of updated NICE guidance. BMJ 2013;346:f2866.

67 Thompson MJ, Ninis N, Perera R, et al. Clinical recognition of meningococcal disease in children and adolescents. Lancet 2006;367:397-403. 